\title{
Comparative Analysis of Higher Education Websites Belonging to Pakistan and Abroad
}

\author{
Paras Chandio ${ }^{1, *}$, Shah Zaman Nizamani ${ }^{1}$, Abbas Ali Ghoto ${ }^{2}$, \\ RafiaNaz Memon ${ }^{1}$ and Mohammad Ali Soomro ${ }^{3}$ \\ 1Department of Information Technology, Quaid-e-Awam University, Pakistan; paraschandio931@gmail.com, \\ shahzaman@quest.edu.pk, rafia@quest.edu.pk \\ 2Department of Mathematics and Statistics, Quaid-e-Awam University, Pakistan; ghotoabbas@hotmail.com \\ 32Department of Computer Systems Engineering, Quaid-e-Awam University, Pakistan; Kalakot2@gmail.com
}

\begin{abstract}
Background: Access to information is made easy by web-based technologies. Higher education institutes take benefits from web-based technologies by receiving and sharing information with students and staff members. The success of a website depends upon many factors, such as usability, type of information, processing speed, and search engine optimisation (SEO). Objective: The quality of higher education institute websites varies, i.e., the websites perform better in some parameters, while they are weak in other parameters. In this research, a comparative analysis is done on the basis of parameters related to speed and SEO of higher education institute websites belonging to Pakistan and other countries. Method: Different parameters related to speed (such as page loading time, connection time, etc.) and SEO are explored with the help of different tools such as Website Grader and Pingdom. Findings: This study shows that the websites of Pakistan are majorly weak in parameters such as page loading time, responsiveness, and SEO. The websites are also poorly developed. Applications/improvements: Findings of this research are not only helpful in improving the quality of new websites, but old websites can benefit from re-engineering.
\end{abstract}

Keywords: Website Performance, Speed, SEO, Website Loading Time

\section{Introduction}

Websites are generally developed for easy access of information. 1 Different organisations use websites improving their business, i.e., business order and record management is done on the websites. Education institutes also benefit from web-based technologies. Tasks of education institutions such as student registration, course offerings and record management, etc., are performed on the websites.

Large numbers of websites are created every day; some websites are taking care of quality standards, while others are weak in quality. High-quality websites improve user satisfaction levels by quickly providing the required information. To analyse the quality of websites, speed, usability and search engine optimisation (SEO) techniques are the important factors. $\underline{2}$

The speed of website loading can be improved by hosting a website in a high-speed server and using efficient algorithms. All the multimedia content such as images should be optimised for reducing disk space. Multimedia contents such as images with large size will increase page loading time, because more data requires more time to load the contents of the websites.

Besides efficient algorithms and high-speed hosting servers, it is also important that websites should be optimised according to search engine requirements, so that users can be familiar with the websites. For SEO, proper guidelines should be followed. By efficiently using html meta tags for description and keywords of

*Author for correspondence 
the websites and providing social network links, search engine ranking can be improved.

The quality of a website can be measured by analysing visible and invisible factors of a website. Visible areas include functionality and user interface of a website. Invisible areas include website speed, responsiveness and SEO. Majority of the work is done on the visible areas of the websites such as graphical design and user interface. In this research, a comparative analysis is done among the higher education websites belonging to Pakistan, USA, Canada, UK, and Australia. The analysis is done on the basis of different invisible quality areas.

The study is divided into five sections. In section II, literature review is given in the area of website evaluation methods and tools. Research methodology is explained in section III. Results of comparative analysis are discussed in section IV. Finally, conclusion is given in section V.

\section{Literature Survey}

A survey of website evaluation tools is available in ref. $\underline{3}$ The tools used for the survey are SEOptimer, Website Grader and Qualidator. Survey results were obtained by applying the tools on the website www.piit.ac.in. The authors have concluded that these evaluation tools are helpful in improving the quality of websites.

In ref. 4,23 websites belonging to Jordan have been evaluated with the help of different usability evaluation tools. The results showed that mostly the websites were easy to use and efficient, while a small number of websites was difficult to use. The researchers have concluded that user interface of the websites should be improved.

Some usability techniques for the evaluation of websites are presented in ref. $\frac{5}{\text { Th }}$ Thesearch suggests that usability techniques such as heuristic evaluation, cognitive walkthrough and focus groups are very useful for improving the quality of websites. The author has also suggested that before evaluation, some information about evaluation need to be gathered regarding time and cost limitations.

Websites of hospitals have been evaluated in ref. $\underline{6}$ using three parameters, which were security, accessibility and usability, with the help of WCAG 2.0 guidelines. The researchers performed this study on 397 hospital websites of famous cities of India. The researchers have found that average size of page is $209502 \mathrm{~kb}$ and average image size is $579 \mathrm{~kb}$. Average page loading time and connection time is 17.23 and 3.9 seconds, respectively. The researchers also found that a majority of websites did not contain multilanguage features. The researchers have concluded that the websites require more security and should also be easy to use.

Usability issues of websites faced by non-technical users are highlighted in ref. $\underline{\underline{Z}}$ For usability testing, two categories of users were selected by the researchers; one category of users was technical and other was nontechnical. An application was developed for testing the performance of both types of users. The evaluation results showed that $60 \%$ non-technical users perform their task while $100 \%$ tasks were performed by technical users. In this research, the authors have highlighted the importance of user interface of websites.

An empirical analysis of website performance was done in ref. $\underline{8}$ Five parameters were used for evaluation. The parameters were efficiency, engaging, ease of use, effectiveness, and error tolerance. The authors have analysed the five parameters on the websites of the Punjab Government. They concluded that improvements are required in the websites against all the parameters.

In ref. 9 , the quality of websites was measured with the help of tools such as SiteChecker and HTML validator. The results showed that for attracting more users, the quality of websites should be improved. A report $\underline{10}$ highlighted that good-quality websites improve business performance. The researchers have given conclusions based upon the data collected from 156 online users.

One report $\underline{11}$ highlighted seven parameters for analysing the performance of e-commerce websites. The parameters were ease of use, usefulness, navigational challenge, website design, content quality, trust and enjoyment. For this study, data were collected from 455 users. The researchers concluded that trust is important for e-commerce websites. Another report $\frac{12}{12}$ studied e-commerce websites based upon different parameters related to performance and usability. The research highlighted the effect of performance and usability on e-commerce websites.

An investigation $\underline{13}$ studied the performance of websites with the help of Apache JMeter. In the research, performance issues are highlighted in different websites. The researchers suggested that the performance of a website can be improved using JMeter software.

A study $\underline{14}$ discussed the reliability and quality of e-governance websites. For this study, 51 websites of Turkey were analysed for measuring the reliability and 
quality. The results of this study showed that software engineers of e-governance should follow the guidelines for developing a good-quality website. Similar results were reported by ref. $\frac{15}{}$ According to their research, most education-related websites are weak in usability and performance.

In ref. $\frac{16}{}$, the effects of websites on customer retention were studied. For this research, the authors collected data from users, review articles and various databases. They also used different sources such as GoogleScholar, ResearchGate, and ScienceDirect for data collection. This research showed that for successful customer retention and satisfaction, some of the important parameters are credibility, correctness, and learnability. In ref. $\frac{17}{\text {, the }}$ researchers developed a framework for analysing the quality of e-commerce websites.

\section{Research Methodology}

The aim of this study was to assess the performance of Pakistani websites with respect to speed and SEO. In order to achieve this aim, a list of countries and a selection of their websites were created. Then a list of parameters was created for analysing the performance of these websites. In the end, different evaluation tools were selected and used for collecting the information based on the selected parameters.

\subsection{Scope and Limitations}

For this research, only websites of higher education institutions were taken. The websites belonged to five different countries. Ten websites were randomly selected from each of the five countries. The countries addressed were:

(i) Pakistan

(ii) USA

(iii) UK

(iv) Australia

(v) Canada

\subsection{Parameters for the Research}

For this research, eight parameters were selected for the comparative analysis of websites. The list of parameters is shown in Table 1.
Table 1. List of parameters

\begin{tabular}{|l|l|}
\hline S. no. & Parameter \\
\hline 1 & Page loading time \\
\hline 2 & Page size \\
\hline 3 & Connection time \\
\hline 4 & Time to receive first byte \\
\hline 5 & Start render time \\
\hline 6 & SEOe \\
\hline 7 & Responsiveness \\
\hline 8 & Page errors and warnings \\
\hline
\end{tabular}

\subsection{Data Collection}

For data collection, freely available website analysis tools were used. Different evaluation tools were used because a single tool cannot provide all the information regarding all the parameters. The tools used were Website Grader, Webpage Test, Pingdom, and Byte Check.

For analysing the SEO, four sub-parameters were selected. The parameters were meta keywords, meta description, heading tags and alt tags. All the information of SEO parameters was manually collected.

\section{Results and Discussion}

After collecting information based on all the parameters, they were analysed with the help of Microsoft Excel. The results of each parameter are separately discussed in the following sections.

\subsection{Page Loading Time}

The average loading time of the websites is given in Figure 1. The slowest page loading time was noted for the websites

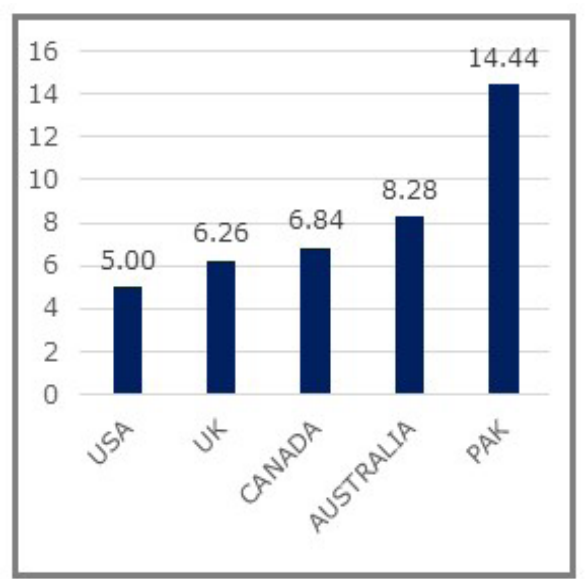

Figure 1. Page loading time. 
of Pakistan, which is 14.44 seconds. While the websites of USA showed the fastest page loading time, which is 5.0 seconds. This suggests that improvements are required in Pakistani websites with respect to page loading time.

\subsection{Page Size}

The average page size of the websites is shown in Figure 2. The largest page size was found for the websites of Australia, which is $1.879 \mathrm{Mb}$, and the least page size was seen for the websites of UK, which is $0.941 \mathrm{Mb}$. The websites of Pakistan were on average $1.012 \mathrm{Mb}$. This performance is satisfactory with respect to page size.

\subsection{Connection Time}

The average connection time for the websites is given in Figure 3. The highest connection time is taken by the websites of Australia, which is 517.99 milliseconds, and the lowest connection time is taken by the websites of USA, which is 55.95 milliseconds. The average connection time is also high for the websites of Pakistan, which is 438.60 milliseconds. This suggests that Pakistani websites should be hosted in a high-speed server.

\subsection{Time to Receive First Byte}

The results of time to receive first byte are given in Figure 4. The highest time is required by the websites of Pakistan, which is 1593.6 milliseconds, while the lowest amount of time is required by the websites of USA, which is 672.56

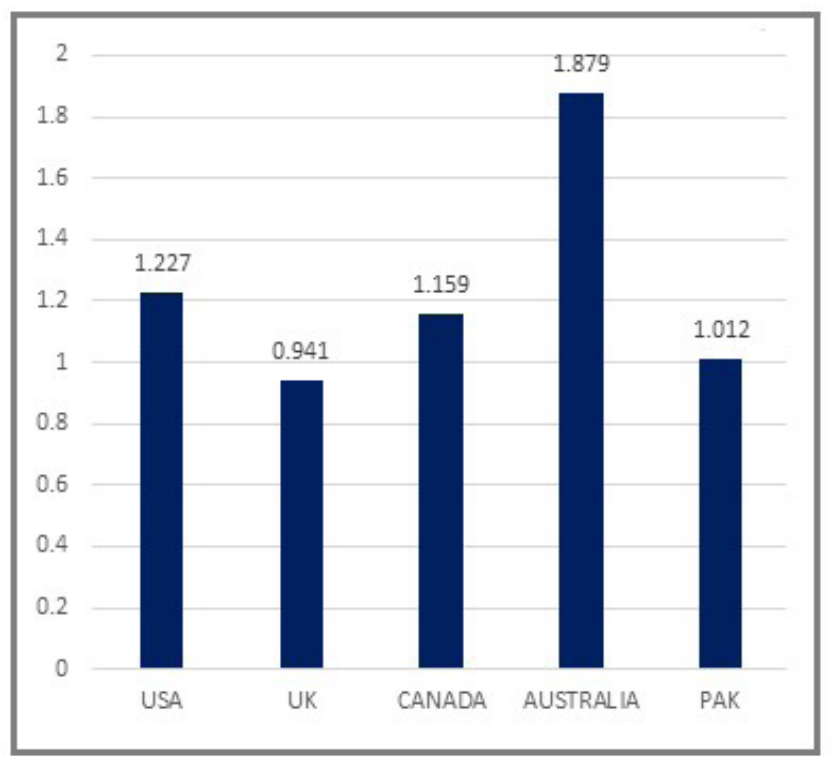

Figure 2. Page size.

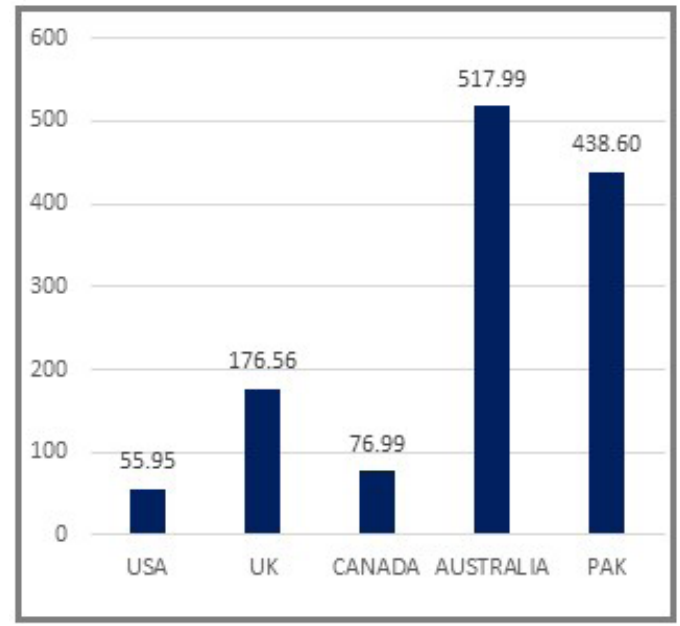

Figure 3. Connection time.

milliseconds. Therefore, improvements are required in the websites of Pakistan for this parameter.

\subsection{Start Render Time}

The average start render time for all the websites is given in Figure 5. The highest start render time is taken by websites of Australia, which is 3.75 seconds, while the lowest start render time is taken by the websites of USA,

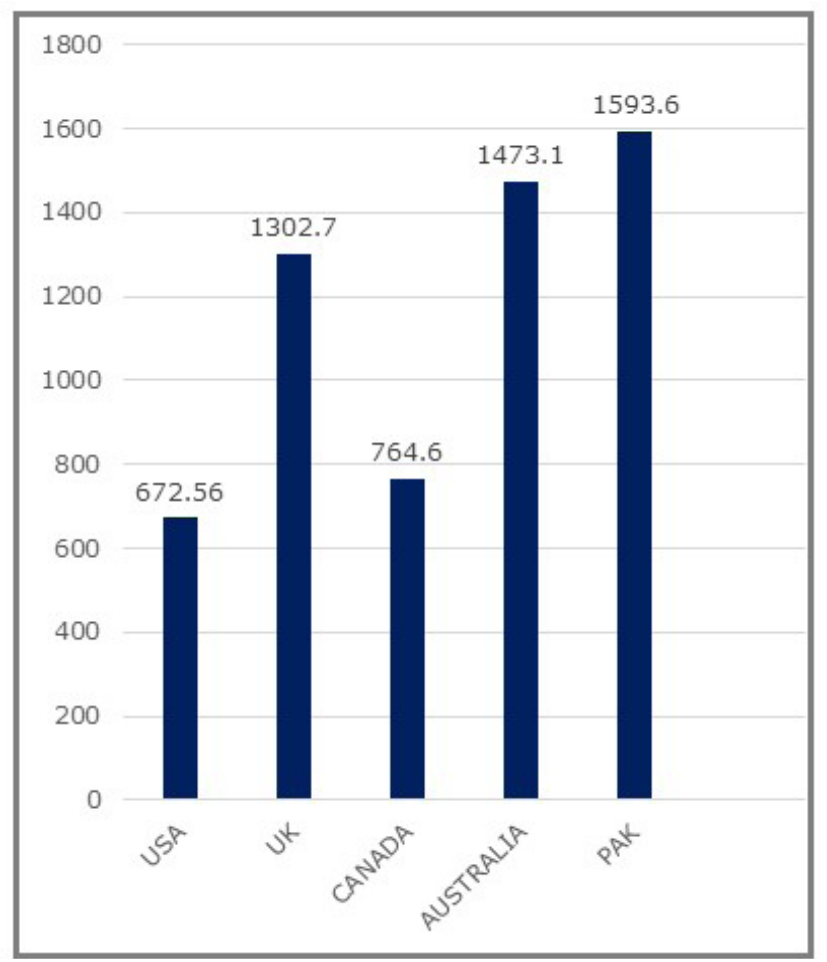

Figure 4. Time to receive first byte. 


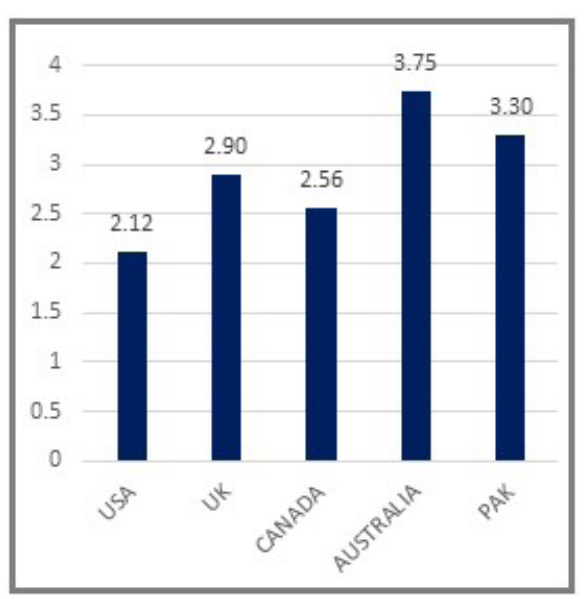

Figure 5. Start render time.

which is 2.117 seconds. However, the average start render time of Pakistani websites is 3.3 seconds. This suggests that higher education websites of Pakistan provide satisfactory performance with respect to start render time.

\subsection{Responsiveness}

Websites should be automatically adjusted for different screen sizes. For this purpose, responsiveness of websites was analysed. The results of responsiveness are shown in Figure 6. Accordingly the websites of USA, UK, Canada and Australia are $100 \%$ responsive, but only $20 \%$ websites of Pakistan are responsive. Therefore, large improvements are required in the area of responsiveness for Pakistani websites.

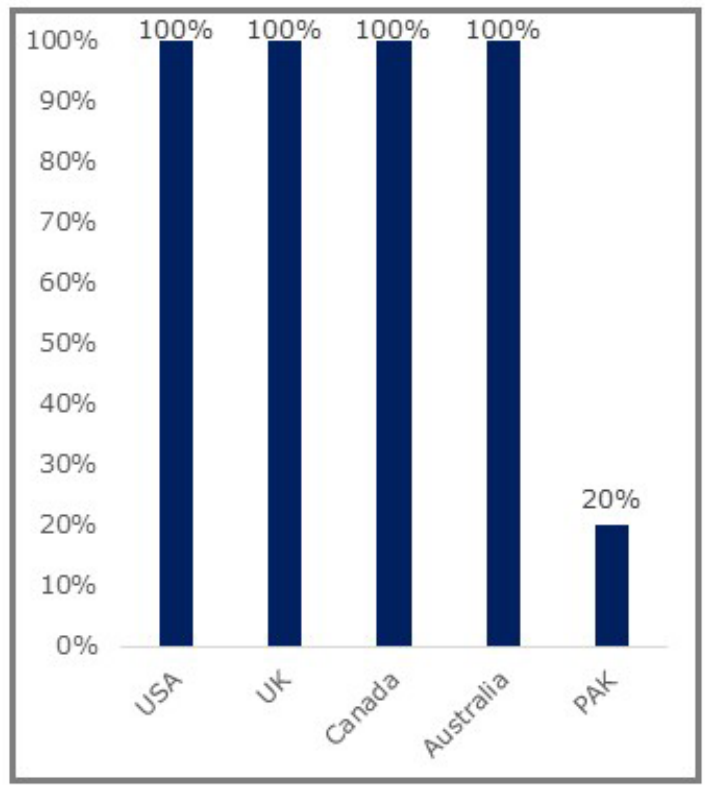

Figure 6. Responsiveness.

\subsection{Page Errors and Warnings}

Websites contain page errors and warnings when proper coding standards are not followed. The results of page errors and warnings for all the websites are shown in Figure 7. The websites of Pakistan contain 16.9 average warnings and 174.4 average errors. Warnings and errors affect the usability and speed of websites; therefore, proper coding standards are not followed in the websites of Pakistan.

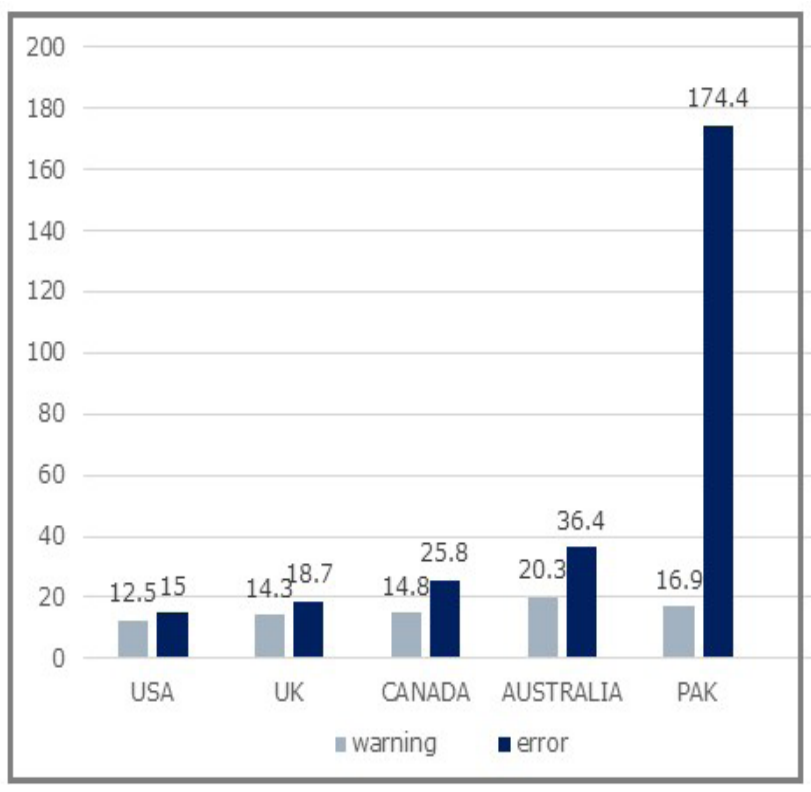

Figure 7. Page warnings and errors.

\subsection{Search Engine Optimisation}

In order to analyse SEO features of websites, data were collected for four parameters of SEO, which were meta keywords, meta description, heading tags and alt tags. Results of all the parameters are separately presented in the following sections.

1) Meta keywords: Meta keywords are very important for SEO. The results of meta keywords used in the websites are given in Figure 8. All the websites of UK contain meta keywords, while only $50 \%$ websites of Pakistan contain meta keywords. Therefore, meta keywords should be included in all the websites of higher education institutes.

2) Meta description: Results of meta description are given in Figure 9. All the websites of USA and UK contain meta description, while only $70 \%$ websites of Pakistan contain meta description. Therefore, 
improvements are required in the area of meta description for Pakistani websites.

3) Heading tags: The results of heading tag analysis are shown in Figure 10. All the websites of UK contain heading tags, while only $50 \%$ websites of Pakistan contain heading tags. Therefore, this area is also in need of improvement.

4) Alt tags: For image searching, alt tags are important. The analysis results are given in Figure 11. All the websites of USA, UK, Canada, and Australia contain image description in alt tags, while only $90 \%$ Pakistani websites contain image description in alt tags. Therefore, some improvement is also required in this regard.

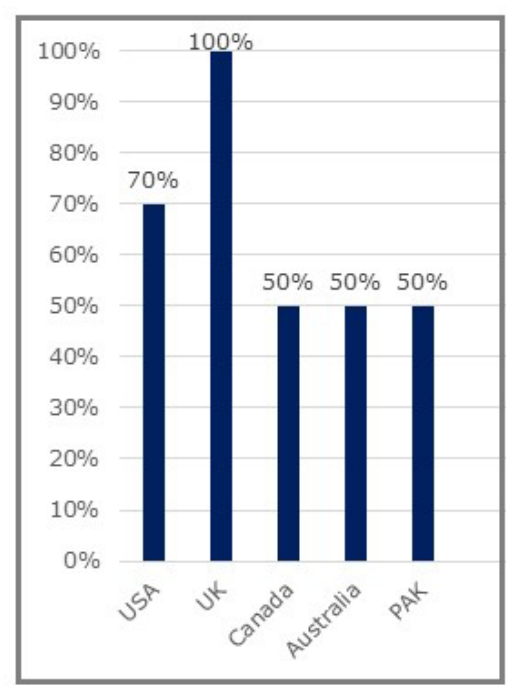

Figure 8. Meta keywords.

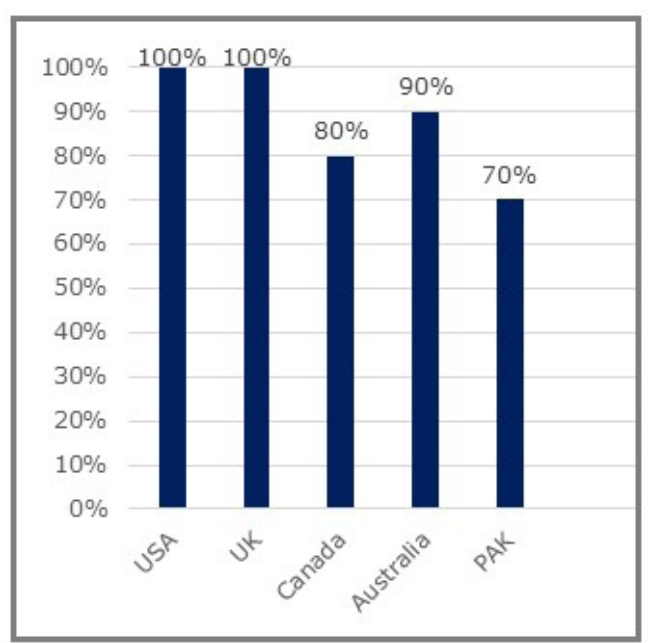

Figure 9. Meta description.

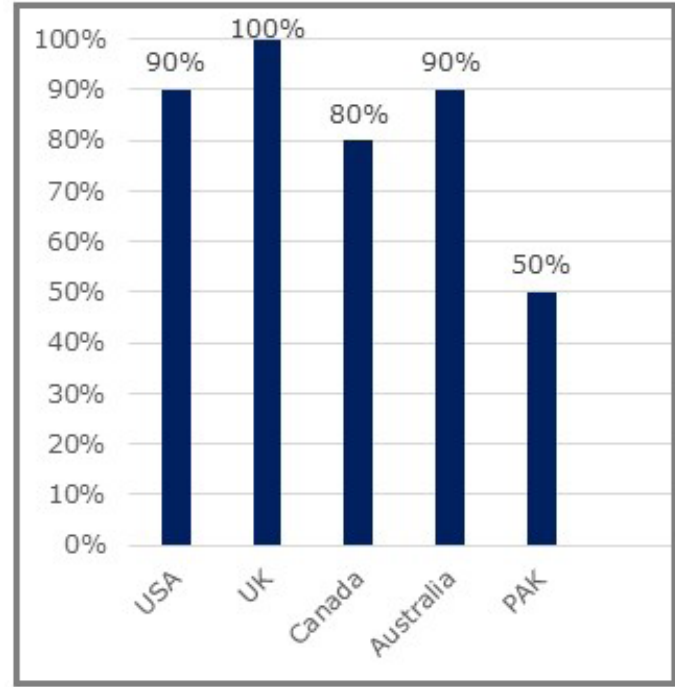

Figure 10. Heading tags.

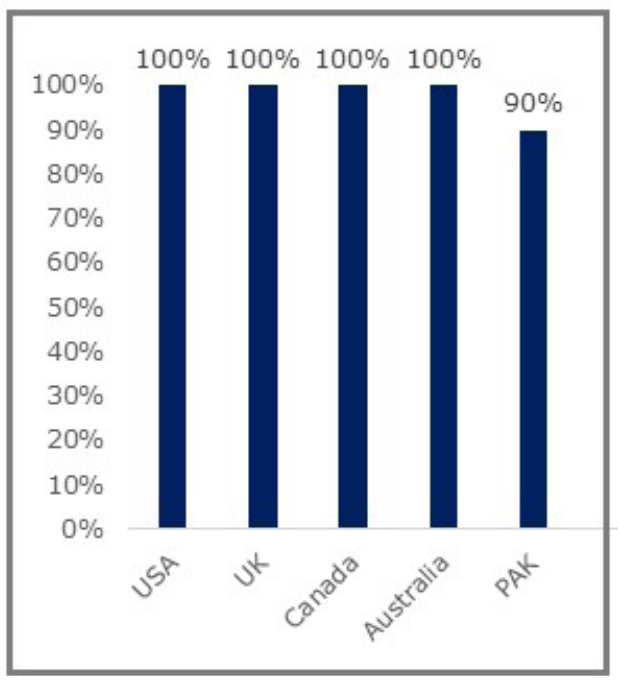

Figure 11. Alt tags.

\section{Conclusion}

In this study, country-wise performance of university websites is analysed using different evaluation tools. The results show that a majority of higher education institute websites of Pakistan are weak with respect to responsiveness, connection time and page loading time. Higher education institute websites of Pakistan are also weak with respect to SEO. Social networking links are also missing in the higher education websites of Pakistan. Improvements are also required in writing the HTML code. Alt tag for images is also not commonly used in the websites of Pakistan. Time to receive first byte and 
connection time can be improved by better server hosting. A dedicated high-speed server can improve performance with respect to both parameters.

\section{References}

1. Nagpal R, Mehrotra D, Bhatia PK. Task based effectiveness evaluation of educational institute websites. In: 2016 international conference on computational techniques in information and communication technologies (ICCTICT); 2016. P. 315-9.

2. Jasim Al-Shujairi YB. What, which and where: examining self-mention markers in ISI and Iraqi local research articles in applied linguistics. Asian Engl. 2018: 1-15.

3. Khandare SS, Gawade S, Turkar V. Survey on website evaluation tools. In: 2017 international conference on recent innovations in signal processing and embedded systems (RISE); 2017. P. 608-15.

4. Najadat H, Al-Badarneh A, Al-Huthaifi R, Abo-Zaitoon A, Al-Omary Y. Evaluating Jordanian universities' websites based on data envelopment analysis. In: 2017 8th international conference on information and communication systems (ICICS); 2017. P. $159-64$.

5. Liu F. Usability evaluation on websites. In: 2008 9th international conference on computer-aided industrial design and conceptual design; 2008. P. 141-4.

6. Kaur A, Dani D, Agrawal G. Evaluating the accessibility, usability and security of hospitals websites: an exploratory study. In: 2017 7th international conference on cloud computing, data science $\&$ engineering-confluence; 2017. P. 674-80.

7. Ijaz T, Andlib F. Impact of usability on non-technical users: usability testing through websites. In: 2014 national software engineering conference; 2014. P. $37-42$.
8. Kaur S, Kaur K, Kaur P. An empirical performance evaluation of universities website. Int J Comp Appl. 2016;146(15):10-16.

9. Kaur S. An automated tool for web site evaluation. Int J Comp Sci Inf Technol. 2012;3(3):4310-13.

10. Lee Y, Kozar KA. Investigating the effect of website quality on e-business success: an analytic hierarchy process (AHP) approach. Decis Support Syst. 2006;42(3):1383-401.

11. Dickinger A, Stangl B. Website performance and behavioral consequences: A formative measurement approach. J Bus Res. 2013;66(6):771-7.

12. Singh T, Malik S, Sarkar D. E-commerce website quality assessment based on usability. In: 2016 international conference on computing, communication and automation (ICCCA); 2016. P. 101-5.

13. Putri MA, Hadi HN, Ramdani F. Performance testing analysis on web application: Study case student admission web system. In: 2017 international conference on sustainable information engineering and technology (SIET); 2017. P. 1-5.

14. Akgul Y. Web site accessibility, quality and vulnerability assessment: a survey of government web sites in the Turkish republic. J Inf Syst Eng Manag. 2016;1(4):50.

15. Wahyuningrum T, Rokhman N, Musdholifah A. Algorithm comparison performance in assessing the quality of university websites. In: 2017 4th international conference on new media studies (CONMEDIA); 2017. P. 19-24.

16. Mkpojiogu EO, Aghahowa MI. The role of website pleasure on customers retention: a systematic review. Comp Softw Media Appl. 2018:1-15.

17. Moretti A, Mason MC, Fatta DD. Measuring website quality: theoretical framework and practical implications for the agro-food sector in the European alpine area. I J Electron Market Retail. 2017;8(3):212-31. 